\title{
Effect of intravitreal dexamethasone implant on macular area parameters in central retinal vein thrombosis
}

\begin{abstract}
This study presents morphological and functional parameters of visual organ in 5 patients with post-thrombotic macular edema against the background of administration of Ozurdex intravitreal dexamethasone implant. 1 month after intravitreal dexamethasone implant administration significant improvement in best corrected visual acuity (BCVA), overall light sensitivity of the central area of the retina, reduction of average thickness of the macular area of the retina as a result of macular edema reduction was marked. In 12 months after treatment some decline in these morphological and functional parameters (except BCVA) with respect to the results after 1 month was reported, but with parameters more close to normal in comparison with that before the treatment.
\end{abstract}

Keywords: retinal vein thrombosis, dexamethasone implant, ozurdex, macular area, edema, morphofunctional parameters
Volume 7 Issue 5 - 2017

\author{
Mukharram M Bikbov, Rinat R \\ Fayzrakhmanov, Inga I Arslangareeva, Timur R \\ Gilmanshin \\ Department of Vitreoretinal and Laser Surgery, Ufa Eye \\ Research Institute, Russia
}

\begin{abstract}
Correspondence: Inga I Arslangareeva, Department of the Vitreoretinal and Laser Surgery, Ufa Eye Research Institute, Address Ufa Eye Research Institute, Pushkin str. 90, Ufa, 450008, Russia, Tel +7 (987) 257-I2-32, +7 (347)272-65-62, Email inga.gilyazova@gmail.com
\end{abstract}

Received: September 22, 2017 | Published: November 07, 2017
Abbreviations: BCVA, best corrected visual acuity; CRV, central retinal vein; $\mathrm{GC}$, glucocorticoids drugs; IOP, intraocular pressure; ME, macular edema; OCT, optical coherence tomography

\section{Introduction}

Thrombosis of central retinal vein (CRV) and its branches in the structure of vascular pathology takes the second place after diabetic retinopathy in frequency of occurrence, severity and functional prognosis causing partial or complete loss of vision. According to experts 16.4 million cases of CRV thrombosis and its branches are registered annually all over the world. ${ }^{1-5}$ Despite significant progress in treatment of retinal vein thrombosis, a macular edema (ME) is a common reason for the central vision decrement in patients with this pathology. In $60-100 \%$ of cases the retinal vein thrombosis is complicated by development of post-thrombotic ME. In case it lasts for three months it may lead to secondary progressive chorioretinal dystrophy accompanied by an irreversible decrease in central vision. ${ }^{4,5}$

The inflammatory response, which also leads to blood-retinal barrier disorder, is one of the main factors contributing to the development of post-thrombotic ME. In particular, an increase in concentration of proinflammatory cytokines IL-1, IL-6, TNF- $\alpha$ and VEGF in the tissue of the eyeball testifies to the fact of inflammation involvement in the development of this condition. ${ }^{6}$ The prevalence of retinal vein occlusion diseases requires development of treatment methods with intraocular drug administration and creation of high therapeutic concentrations of medications in the inner membranes of the eye. The drug administration directly into the vitreous cavity have a number of advantages associated with its intraocular concentration increase and more prolonged impact directly on the lesion, as well as a significant decrease in systemic side effect of the drug applied. ${ }^{7}$

Intravitreally injected drugs with a pronounced anti-edematous effect, including in retinal vein thrombosis comprise anti-VEGF (vascular endothelial growth factor) drugs and glucocorticoids drugs (GC). The mechanism of anti-vasoproliferative drugs based on the inhibition of effects of vascular endothelial growth factor in tissues of the eye significantly decreasing vascular permeability and contributing to macular edema and pathological angiogenesis process reduction. ${ }^{8,9} \mathrm{GC}$ is natural biologically active substances that play an important role in the activity of many life forms. They have strong anti-inflammatory, anti-allergic, immunosuppressive properties and are actively involved in metabolism regulation. ${ }^{10,11}$ In this regard, currently intravitreal administration of sustained release glucocortico steroids is one of directions in ME treatment. Intravitreal triamcinolone acetonide (Kenalog) is widely used. Studies have shown that even at a single administration of the drug a significant reduction in edema and retinal thickness was observed according to optical coherence tomography (OCT). In some patients there was an increase in visual acuity, and a similar effect of a single application may last up to 3-6 months. ${ }^{12}$ According to some authors, with the drug absorption and its concentration decrease in the vitreous cavity, its effect decreases and retinal macular edema grows again. ${ }^{6,7}$

The half-life period of triamcinolone acetonide from the vitreous body lasts from 1.6 to 18.6 days, and the period of the drug presence in the vitreous cavity - from 21 to 140 days. ${ }^{13}$ Researchers have identified three stages of the triamcinolone acetonide effect on the retina thickness: a rapid decrease in edema, condition stabilization and ME recurrence. ${ }^{12}$ As a rule, eventually all patients with ME will need repeated injections of the drug. In addition, the results of clinical studies of Kenalog intravitreal injection safety testify to side effects of the drug. These include an increase in intraocular pressure (IOP) with possible subsequent development of glaucoma, posterior subcapsular cataract formation..$^{14,15}$ New medical and surgical methods give hope for the best results of thrombotic-related maculopathy treatment. One of the attempts to reduce frequency and severity of GC side effects -is development of sustained release implants, which are also injected into the vitreous body. They contain the drug which is effused in small 
portions over a period of several months. Due to this mechanism, relatively low concentration of active substance is present in the vitreous cavity, which may reduce a cataractogenic effect and will not increase the IOP.

Intravitreal implants containing dexamethasone are introduced into the eye cavity via a specially designed injector and are used for ME therapeutic treatment, developed, for example, as a result of CRV occlusion or its branches. Dexamethasone is a potent GC, capable of suppressing inflammation, decreasing the severity of edema, capillary permeability, fibrin deposition and migration of phagocytes to the area of inflammation. Moreover, the drug inhibits vascular endothelial growth factor, prevents the release of prostaglandins, which are in particular ME mediators. The implant is made of a solid polymer that undergoes biodegradation in the vitreous cavity. ${ }^{16,17}$ However, to date there is no consensus on the effectiveness of long-term use of intravitreal dexamethasone implant in treatment of post-thrombotic ME.

\section{Purpose}

To investigate functional and morphometric parameters of the central area of the retina with post-thrombotic ME on the background of intravitreal dexamethasone implant administration.

\section{Materials and methods}

It was examined 5 patients ( 5 eyes) with newly diagnosed ME complicated CRV thrombosis (study group). Of these, 4 were men, 1 - woman. The mean age of patients was 55,8 $\pm 3,65$ years. The duration of the disease since the appearance of specific complaints before the survey ranged from 2 weeks to 7 months. All patients received the standard course of conservative therapy in the community, which was not followed by a significant improvement in visual function. Among co-existing diseases 3 patients $(60.0 \%$ of cases) had hypertension, 1 $(20.0 \%)$ - chronic glomerulonephritis, $1(20.0 \%)$ - chronic leukemia.

The control group consisted of 5 people (10 eyes) aged 51 to 68 years (average 59,14 $\pm 3,14$ years), who had no eye diseases except for presbyopia. The group was comparable to the performance status of the study group (4 patients had hypertensive disease, 1 patient chronic pyelonephritis).

Standard ophthalmic examination included: best corrected visual acuity (BCVA), ophtalmobiomicroscopy using a spherical lens of
78 diopters, Fluorescein angiography (FAG) using fundus camera (Visucam-500), optical coherence tomography (Retina Scan - 3000, Nidek Technologies). All patients in the study group had single intravitreal injection of the Ozurdex implant in the operating room according to the standard procedure using the original injector to form a scleral tunnel with $22-\mathrm{G}$ needle. Examination and IOP monitoring was performed in an hour, day, month, and a year after surgery. The maximum follow-up period after intravitreal intervention was 12 months. Statistical analysis was performed using Microsoft Excel 97, Statistica 6.1 applications. Differences were considered statistically significant at $\mathrm{p}<0.05$.

\section{Results}

Patients in the control group had $0,92 \pm 0,3$ BCVA. According to microperimetry overall light sensitivity of the central part of retina at 45 points of the displayed pattern was equal to $18,64 \pm 1,32 \mathrm{~dB}$. According to the OCT data, a correct macular profile with a fovea in the centre and retinal thickness ranging from $221,4 \pm 10,97$ to $355,2 \pm 12,17$ micron was observed. Before intravitreal Ozurdex injection patients of the study group had significantly reduced BCVAan average of $0,09 \pm 0,03$. Ophthalmoscopically changes typical to CRV thrombosis in the form of tarnish, turbidity of edematous retina, lack of macular reflex, venous varix and tortuosity, fogging of their contours, hemorrhages in various stages of resorption, protrusion in the macula were observed. FAG results showed that 4 people $(80.0 \%$ of cases) had ischemic type of CRV thrombosis, 1 patient $(20.0 \%)$ had non-ischemic type of CRV thrombosis.

According to microperimetry overall light sensitivity of the central area of the retina before the specific treatment was significantly reduced and amounted to $3,18 \pm 0,19 \mathrm{~dB}$. The average thickness of the retina in the macular area resulting from edema increased by 1.3 times and at all points of the examined area ranged from $247.86 \pm 39,06$ to $494.07 \pm 40,22$ micron with gradual thickening from the periphery to the center. As seen from Table 1, in patients of the study group the total thickness of the retina was significantly higher than in the control group ( $<<0.001)$. Differences in the average thickness of the fourth layered area of the retina between the two groups were statistically reliable ( $\mathrm{p}<0.05$ ), and of the $1 \mathrm{st}$, 3rd, 5th zones - highly reliable ( $<0.001$ ). In the studied group it should be particularly marked 1.5 fold increase of the third area thickness covering the outer plexiform layer and the inner nuclear layers and 4-fold increase of the 5th zone consisting of nerve fiber layer and the inner limiting membrane.

Table I Average thickness parameters of the central retinal area zones in patients of the study (before treatment) and control groups, M $\pm m$, micron

\begin{tabular}{llll}
\hline Layered Areas of the Retina & Study Group (n=5) & Control Group (n=10) \\
\hline Areas & Overall Thickness & $386,97 \pm 16,26^{* *}$ & $299,01 \pm 7,56$ \\
1 & Retinal pigment epithelium - outer segments of photoreceptors & $63,26 \pm 1,01 * *$ & $72,15 \pm 0,81$ \\
2 & Inner segments of photoreceptors - Outer nuclear layer & $85,78 \pm 4,48$ & $76,77 \pm 2,93$ \\
3 & Outer plexiform layer - inner nuclear layer & $94,94 \pm 5,08^{* *}$ & $62,11 \pm 4,08$ \\
4 & Inner plexiform layer - Ganglion cell layer & $88,05 \pm 5,54^{*}$ & $69,82 \pm 4,23$ \\
5 & Nerve fiber layer - inner limiting membrane & $54,6 \pm 3,26^{* *}$ & $13,76 \pm 1,4$ \\
\hline
\end{tabular}

* - Significant differences compared to the control group $(p<0,05), * *$ - $p<0,00$ I 
A month after intravitreal Ozurdex injection subjectively patients began to notice vision improvement. BCVA increased to an average of $0.19 \pm 0.05$. The results of the overall light sensitivity of the central area of the retina also had a positive dynamics and amounted to $11,07 \pm 0,97$ $\mathrm{dB}$, which is 3.5 times higher than before the treatment. OCT revealed a decrease in macular edema in 1.3 times with fluctuations in values from $234,5 \pm 68,89$ to $333,75 \pm 35,46$ micron. The macula profile acquired the right shape with a fovea in the centre (Figure 1), where the thickness of the retina in fovea averaged $273,75 \pm 36,65$, which is 1.5 times less than that before treatment $(425,36 \pm 57,87$ micron, $\mathrm{p}<0,05)$, and was comparable to the parameters of the control group $(221,4 \pm 57,87$ micron). ME reduction in patients with CRV thrombosis a month after intravitreal Ozurdex injection was due to a moderate thickness reduction of all layered areas of the retina (Table 2), but especially significant positive changes affected areas of inner segments of photoreceptors, outer nuclear, outer plexiform and inner nuclear layers.

\section{Macula profile changes}

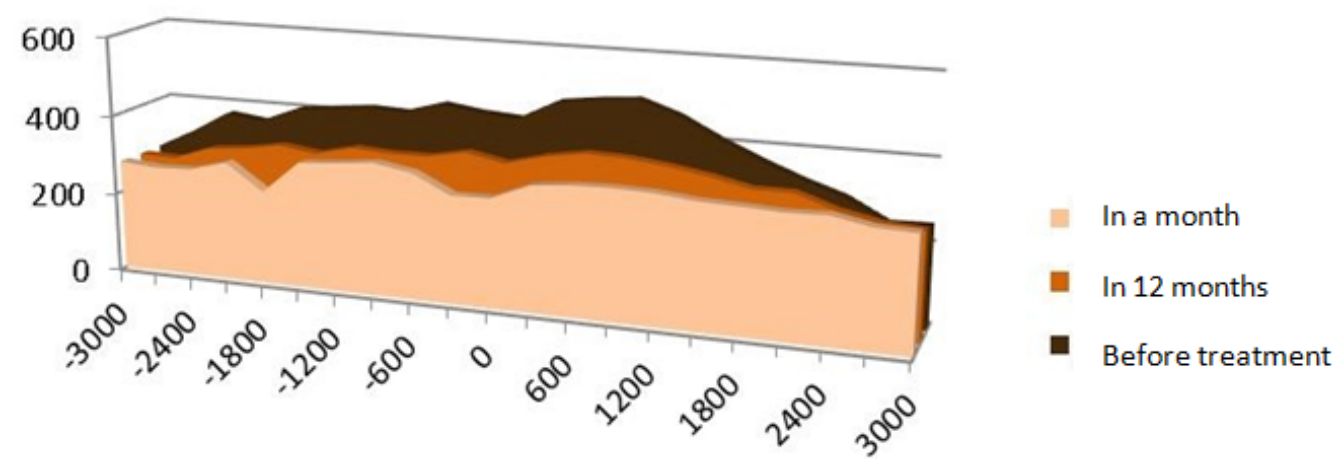

Horizontal section of macula, micron

Figure I Scheme of change in thickness and profile of the macular area in patients with post-thrombotic macular edema before treatment and at various times after Ozurdex intravitreal injection.

Table 2 Average thickness parameters of the central retinal area in patients with post-thrombotic macular edema before treatment and at various times after intravitreal Ozurdex injection, $\mathrm{M} \pm \mathrm{m}$, micron

\begin{tabular}{|c|c|c|c|c|}
\hline \multicolumn{2}{|c|}{ Layered Areas of the Retina } & \multirow{2}{*}{$\begin{array}{l}\begin{array}{l}\text { Before Treatment } \\
(n=5)\end{array} \\
386,97 \pm 16,26\end{array}$} & \multirow{2}{*}{$\begin{array}{l}\text { In a Month after } \\
\text { Treatment }(\mathbf{n = 5}) \\
299,65 \pm 5,48^{* * * *}\end{array}$} & \multirow{2}{*}{ 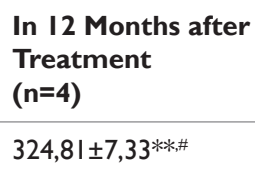 } \\
\hline & Overall thickness & & & \\
\hline 1 & $\begin{array}{l}\text { Retinal pigment epithelium - outer } \\
\text { segments of photoreceptors }\end{array}$ & $63,26 \pm I, 01$ & $58,5 \pm 1,45^{*}$ & $62,97 \pm 1,07^{\#}$ \\
\hline 2 & $\begin{array}{l}\text { Inner segments of photoreceptors - Outer } \\
\text { nuclear layer }\end{array}$ & $85,78 \pm 4,48$ & $60,61 \pm 1,64 * * *$ & $67,6 \pm 2,05 * * * \ldots \#$ \\
\hline 3 & Outer plexiform layer - inner nuclear layer & $94,94 \pm 5,08$ & $70,6 \pm 2,49 * *$ & $77,97 \pm 4,27^{*}$ \\
\hline 4 & Inner plexiform layer - Ganglion cell layer & $88,05 \pm 5,54$ & $63,1 \pm 3,57 * *$ & $69,1 \pm 3,4^{* * *}$ \\
\hline 5 & $\begin{array}{l}\text { Nerve fiber layer - inner limiting } \\
\text { membrane }\end{array}$ & $54,6 \pm 3,26$ & $45,54 \pm 2,57^{*}$ & $43,3 \pm 2,42 *$ \\
\hline
\end{tabular}

$*-\mathrm{p}<0,05, * *-\mathrm{p}<0,01, * * * \mathrm{p}<0,001$ (the differences were significant in comparison with values prior to treatment)

$\#-p<0,05, \# \#-p<0,01$ (the differences were significant in comparison with values in a month after treatment)

In the long-term follow-up (12 months), the following results were obtained. BCVA averaged $0,21 \pm 0,14$. According to the microperimetry the overall light sensitivity of the central area of the retina was equal to $4,8 \pm 0,76 \mathrm{~dB}$, which was slightly above the similar parameters prior to the treatment, however, significantly lower than the results in a month after the intravitreal dexamethasone implant injection. OCT showed a change in the macula profile in the form of foveolar flattening (Figure 1). The average thickness of the retina at all points of the study area increased (varying from $273 \pm 19,04$ to $376,67 \pm 3,56$ micron). Its thickness in the fovea became greater 1.5 times compared to the control group, and 1.2 times in comparison with the results obtained one month after the Ozurdex injection, which was, however, significantly lower than prior to the treatment. ME relapse in patients with CRV thrombosis in a year after treatment has 
arisen due to a moderate increase in thickness of the zones of pigment epithelium, inner and outer segments of photoreceptors, outer nuclear layer (Table 2). During the entire period of the study no significant increase in the IOP level was observed, optical media transparency of lens maintained.

\section{Discussion}

This work is devoted to treatment of macular edema in the retinal vein occlusion processes being one of the leading causes of a persistent reduction in visual acuity. The article is obviously relevant and timely, given the fact that ME is the main and almost permanent clinical sign of retinal vein occlusion. In this paper it was investigated the effect of dexamethasone implant on morphological and functional parameters of the central area of the retina in patients with CRV occlusion. The concept of this research paper included a study of the dynamic pattern of the retina thickness and its layers, visual acuity and light sensitivity.

The results of this work testify to the fact of significant reduction in thickness of the central area of the retina, recovery of macular interface and fiber retinal zones already within a month after the injection. This study confirms also a significant improvement in functional parameters within the specified period in the form of increased visual acuity and light sensitivity according to microperimetry. Thus, a 1.5fold decrease in macular edema with a 2-fold increase in visual acuity, and 3 -fold increase in light sensitivity in the central area within 1 month of the study were marked by the authors. The results identified in the present study correspond to the GENEVA research, which clearly indicates the time of the greatest efficiency of the implant corresponding to the second month after the start of treatment. The therapeutic effect was observed after the drug administration for 30 days; it remained without negative dynamics within 4 months and persisted for at least 4-6 months. ${ }^{18}$ The maximum efficiency of treatment with dexamethasone implant was marked on $60-90$ day after the injection, while clinical response maintained within 6 months. ${ }^{19}$ The GENEVA protocol recommends the re-injection of the implant in approximately 6 months after the previous administration.

The duration of the study is an extremely important feature of this work, given the scarcity of the published data covering such period. The authors describe the morphological and functional parameters deterioration in 12 months after the implant administration. The studied results were slightly better than similar figures before treatment, but significantly worse than results in a month after intravitreal dexamethasone implant injection. Thus, the authors marked 1.08-fold increase in thickness of the macular area, decrease in visual acuity by 1.1 and the light sensitivity of the central region by 2.3 compared to the parameters after a month of the treatment. This fact is probably due to decrease in concentration of the drug in tissues of the eye because of the time factor. Mayer WJ et al ${ }^{20}$ with fairly extensive clinical material ( 22 patients with central retinal vein thrombosis) give some different data related to both functional and anatomical parameters. Thus, the average visual acuity improved 1.2 times with 2 times decrease in central retinal thickness from 604.4 micron to 301.08 micron compared to the data before the treatment at constant positive dynamics of the disease during the 12-month study period. Thus, it can be said about improvement of anatomical and functional properties of the visual organ, even a year after the start of retinal occlusion processes treatment with sustained-release dexamethasone. However, the degree and dynamic pattern in changes of these parameters should be clarified during the specified period.

\section{Conclusion}

i. Intravitreal Ozurdex injection in patients with central retinal vein thrombosis provides a reduction in post-thrombotic ME, improvement of visual function and increase in light sensitivity of the central area of the retina already in a month after the implant injection.

ii. Positive morphometric changes in the central area of the retina associated with Ozurdex in post-thrombotic ME relate to the inner segments of photoreceptors, outer nuclear, outer plexiform and inner nuclear layers.

iii. The therapeutic effect was observed after 1 year of treatment in spite of absence of consistent positive dynamics in morphofunctional parameters within 12 months of the study.

iv. There was no negative effect of dexamethasone implant on IOP and transparency of the lens within one-year follow-up.

v. Due to the small number of observations it is necessary to continue studies of the intravitreal dexamethasone implant effectiveness in patients with macular edema on the background of the retinal vein thrombosis.

\section{Conflicts of interest}

No conflict of interest.

\section{References}

1. Bikbov MM, Fayzrakhmanov RR, Gil'manshin TR, et al. Modern tendencies of conservative treatment of retinal vein thrombosis. Cataract and refractive surgery. 2014;3(14):11-16.

2. Bikbov MM, Fayzrakhmanov RR, Gil'manshin TR, et al. Functional vision as a complex index of visual function in patients with ophthalmopathology. Medical almanac. 2014;1(31):59-62.

3. Katsnel'son LA, Forofonova TI, Bunin A Ya. Vascular eye diseases. Moscow, Russia, Meditsina, 1990; p. 272.

4. Tankovskiy VE. Retinal vein thrombosis. Moscow, Russia, Voenizdat. $2000 ;$ p. 261 .

5. Tul'tseva SN, Astakhov Yu S, Retinal vein occlusion etiology, pathogenesis, clinical findings, diagnosis and treatment. St. Petersburg: Izdatel'stvo N-L, Russia. 2010; p.112.

6. Shadrichev FE, Shklyarov EB, Grigor'eva NN. The usage of corticosteroids in treatment of diabetic macular edema. Ophthalmic statements. 2011;4(2):76-88.

7. Gil'manshin TR. Intravitreal injection of Kenalog in vitreoretinal surgery Cand Diss (Med Sci). Moscow, Russia. 2008.

8. Bikbov MM, Fayzrakhmanov RR, Yarmukhametova AL. Age-related macular degeneration. Moscow, Russia, Aprel'. 2013; p. 196.

9. Bikbov MM, Babushkin AE, Orenburkina OI. The use of anti-VEGF drugs in treatment of neovascular glaucoma. Annals of ophthalmology. 2012;128(5):50-53.

10. Barkhanova AG. Clinical pharmacology of glucocorticoids and tactics of usage. Moscow, Russia, Ts OLIUV. 1985; p. 23.

11. Vogralik VG. Hormones and hormonal agents in clinical picture of internal diseases. Moscow, Russia, Meditsina, 1974; p. 200.

12. Coleman HR, Yuan P, Kim H . Intravitreal injection of triamcinolone for diffuse macular edema. Arch Ophthalmol. 2004;122(7):1085-1086. 
13. Beer PM, Bakri SJ, Singh RJ, et al. Intraocular concentration and pharmacokinetics of triamcinolone acetonide after a single intravitreal injection. Ophthalmology. 2003;110(4):681-686.

14. Bacri SJ, Beer PM. The effect of intravitreal triamcinolone acetonide on intraocular pressure. Ophthalmic Surg Lasers and Imaging. 2003;34(5):386-390.

15. Park SH, Jaffe GJ, Fekrat S. Intravitreal triamcinolone acetonide in eyes with cystoid macular edema associated with central retinal vein occlusion. Am J Ophthalmol. 2003;136(3):419-425.

16. Mayer WJ, Wolf A, Kernt M, et al. Twelwe-month experience with Ozurdex for the treatment of macular edema associated with retinal vein occlusion. Eye. 2013;27:816-822.
17. Belyy Yu A, Novikov SV, Tereshchenko AV, et al. Delivery mechanisms of medications to structures of posterior eye segment. Cataract and refractive surgery. 2014;14(2):10-14.

18. Haller JA, Bandello F, Belfort R, et al. Dexamethasone intravitreal implant in patients with macular edema related to branch or central retinal vein occlusion twelve-month study results. Ophthalmology. 2011;118:2453-2460

19. Haller JA, Bandello F, Belfort R, et al. Randomized, sham-controlled trial of dexamethasone intravitreal implant in patients with macular edema due to retinal vein occlusion. Ophthalmology. 2010;117(6):1134-1146.

20. Mayer WJ, Wolf A, Kernt M, et al. Twelve--month experience with Ozurdex for the treatment of macular edema associated with retinal vein occlusion. Eye (Lond). 2013;27(7): 816-822. 